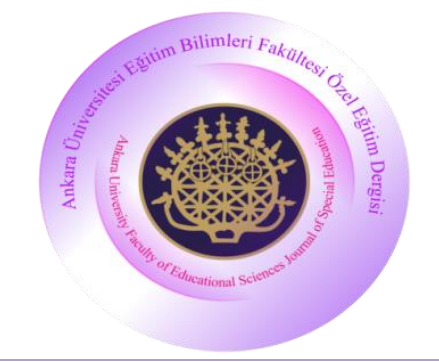

DERLEME

\section{Ankara Üniversitesi Eğitim Bilimleri Fakültesi Özel Eğitim Dergisi}

Yıl: 2018, Cilt: 19, Sayı: 1, Sayfa No: 181-198

DOI: 10.21565/ozelegitimdergisi.279652

\title{
Okul Öncesi Kaynaştırmada Kalite: Kapsam ve Değerlendirme
}

\author{
Betül Yılmaz \\ Gazi Üniversitesi
}

\begin{abstract}
Öz
Kaynaştırma ile ilgili pek çok boyut ülkemiz özelinde ele alındığında bazı soru işaretlerini de beraberinde getirmektedir. Bu bağlamda, eğitim ortamlarında sunulan hizmetlerin kalitesi birçok boyutu etkileyebilecek bir tartışma konusu olarak ortaya çıkmakta ve bu durum okul öncesi kaynaştırmada daha da belirgin hale gelmektedir. Kalite kavramı, hizmetlerin sunumu ve niteliği açısından oldukça önemlidir. Son yıllarda kaynaştırmanın "ne" olduğundan çok, "nasıl” ele alındığı ve nasıl kaliteli hale gelebileceği, bu durumun hangi özellikler bağlamında ele alınacağı uluslararası alanyazında sıklıkla tartışılmaya başlanmıştır. Bu makale kapsamında okul öncesi kaynaştırmanın kalitesi, kalite değerlendirmeyle ilgili boyutları ve bu doğrultuda kullanılabilecek değerlendirme araçları ele alınmaktadır.
\end{abstract}

Anahtar sözcükler: Kaynaştırma, okul öncesi kaynaştırma, kalite, kalite değerlendirme.

\section{Önerilen Atıf Sekli}

Yılmaz, B., \& Karasu, N. (2018). Okul öncesi kaynaştırmada kalite: Kapsam ve değerlendirme. Ankara Üniversitesi Eğitim Bilimleri Fakültesi Özel Eğitim Dergisi, 19(1), 181-198. doi: 10.21565/ozelegitimdergisi.279652

*Sorumlu Yazar: Arş. Gör., E-posta: betulyilmaz@gazi.edu.tr, https://orcid.org/0000-0002-2633-5592

**Doç. Dr., E-posta: necdetkarasu@yahoo.com, https://orcid.org/0000-0001-7507-4109 
Erken çocukluk dönemindeki özel gereksinimli çocuklar ülkemizdeki yasalar ve yönetmelikler çerçevesinde, okul öncesi eğitim kurumlarında normal gelişim gösteren akranları ile birlikte eğitim alabilmektedir. Özel gereksinimli çocukların temel eğitim sınıflarında normal gelişim gösteren akranları ile bir arada olması "kaynaştırma" (inclusion) kavramını gündeme getirmekte ve bu kavram en az kısıtlayıcı ortam temelinde şekillenmektedir (Batu ve Kırcaali-İftar, 2005; Kargın, 2004; Salend, 1998).

Okul öncesi kaynaştırma, okul öncesi eğitim veren okulların diğer okullardan farklı fiziksel özelliklere sahip olması, uygulanan eğitim programının gelişimsel ve çocuk merkezli olması, okul öncesi dönem çocuklarının ilkokul dönemi çocuklarından farklı gelişimsel özellikler taşıması ve akademik başarı odaklı sistemin okul öncesi dönemde yer almaması gibi özelliklerinden dolayı ilkokul ve sonrasındaki kaynaştırma uygulamalarından farklılaşmaktadır (Wolery ve Odom, 2000). Otuz yılı aşkın bir süredir yapılan araştırmalar yetersizliği olan çocukların normal gelişim gösteren akranları ile bir arada bulunmasından sağladıkları yararları ortaya koymuştur (Bruder, 2010; Henninger ve Gupta, 2014). Kaynaştırma ortamında bulunan özel gereksinimli çocukların akademik performansları, sosyal gelişimleri ve davranışları üzerinde kaynaştırma uygulamalarının olumlu etkisi bulunmaktadır (Buysse ve Bailey, 1993; Lipsky ve Gartner, 1996). Örneğin; kaynaştırma programlarında yer alan çocukların dil gelişimleri ve sosyal yeterlikleri artmakta, erken okuryazarlık becerilerinde önemli ilerleme görülmektedir (Green, Terry ve Gallagher, 2014; Rafferty, Piscitelli ve Boettcher, 2003). Bunun yanısıra, bu çocukların akranları ile olumlu sosyal etkileşimleri gelişmekte (Hollingsworth ve Buysse, 2009) ve sosyal kabul düzeyleri de artmaktadır (Odom ve diğ., 2006). Kaynaştırma ortamlarında bulunan çocuklar, ayrıştırılmış ortamlarda eğitim gören benzer özellikteki akranlarına göre bireyselleştirilmiş eğitim programlarında yer alan iletişim, sosyal ve akademik becerileri daha kısa sürede öğrenebilmektedir (Hunt, Farron-Davis, Beckstead, Curtis ve Goetz, 1994). Kaynaştırma uygulamalarının yararları özel gereksinimli çocukların sadece en az kısıtlayıcı ortamda bulunması ile ortaya çıkmamakta; bu çocukların sosyal ilişkilerini destekleme, aktif katılımını sağlama ve tüm çocuklar için öğrenme kazanımlarının artması ile kaynaştırma ortamından beklenen amaçlar karşılanmaktadır (Odom, Buysse ve Soukakou, 2011). Yüksek kaliteli ortamların çocuklara daha fazla davranışsal ve gelişimsel kazanımlar sağlaması (Bryant, Burchinal, Lau ve Sparling, 1994; Burchinal, Roberts, Nabors ve Bryant, 1996; Burchinal ve diğ., 2000); okul öncesi kaynaştırma programlarının değerlendirilmesine, desteklenmesine ve öğretmenlerin profesyonel gelişimine yönelik bakış açısı sağlaması (Soukakou, Winton, West, Sideris, ve Rucker, 2015; Vlachou ve Fyssa, 2016) ve okul öncesi kaynaştırmanın kalite standartlarının oluşturulması bağlamında (Soukakou ve diğ. 2015), kaynaştırmada "kalite" konusu önemli bir boyut olarak karşımıza çıkmaktadır.

Uluslararası alanyazında vurgulandığı üzere, yaygın düşüncenin aksine yetersizliği olan çocuklara kaynaştırma ortamlarında eğitim sağlamanın maliyeti, ayrıştııılmış ortamlardakinden daha pahalı değildir (Barton ve Smith, 2014; Odom ve diğ., 2001). Kaynaştırmanın başarısı, çocuklara ve ailelerine sağladığı kazanımlar, kaynaştırma kapsamında sunulan hizmetler, kaynaştırma ile ilişkili personelin yeterlikleri gibi tartışılan pek çok boyutu olmakla birlikte, hem uzun ve kısa dönemdeki yararları hem de düşük maliyetli uygulama olması, ülkeleri okul öncesi kaynaştırmaya yönlendirmektedir. Bununla birlikte, kaynaştırma uygulamalarının kalitesi alanyazında son yıllarda sıklıkla ele alınan önemli bir odak konu olarak karşımıza çıkmaktadır. Kalite değerlendirmeye yönelik çalışmalar kapsamlı biçimde ele alınmakta, okul öncesi kaynaştırmaya yönelik artan ilgi, sunulan hizmetlerin farklı boyutlardaki göstergeleri ile kalite bağlamında değerlendirilerek okul öncesi kaynaştırma uygulamalarının desteklenmesi gerektiği vurgulanmaktadır (Loreman, Forlin ve Sharma, 2014). Özel gereksinimli çocuklar ve ailelerinin kaynaştırmadan en üst düzeyde faydalanabilmesi için eğitim programlarının düzenlenmesi, geliştirilmesi ve değerlendirilmesi için kaynaştırma uygulamalarının kalitesinin değerlendirilmesi gerekmektedir (Buysse ve Hollingsworth, 2009). Son çeyrek yüzyılda yetersizliği olan çocukların kaynaştırılması ile ilgili yapılan çalışmalara bakıldığında kaynaştırma uygulamalarını etkileyen iki yönelimden birinin kalite değerlendirme olduğu görülmektedir (Odom ve diğ., 2011). Türkiye'de ise okul öncesi kaynaştırma ortamlarının kalitesi yeni gündeme gelmeye başlayan bir konudur. Bu makalenin amacı, okul öncesi kaynaştırmanın kalitesi, kalite değerlendirmenin boyutları ve bu amaçla kullanılabilecek değerlendirme araçları hakkında bilgi sunarak konu ile ilgili bakış açısı sağlamaktır. 


\section{Okul Öncesi Kaynaştırmanın Kalitesi}

Betimsel ve normatif özellikleri olan bir kavram olarak tanımlanan kalite, pek çok açıdan ele alınabilmekte (Adams, 1993); kalite kavramı farklı gruplara göre farklı şekillenmektedir. Çocuklar, aileler, işverenler ve diğer hizmet sağlayıcılar farklı değerler ve ihtiyaçları temelinde kaliteyi farklı biçimde tanımlamaktadır (Katz, 1993; Layzer ve Goodson, 2006). Ancak "kalite" genel olarak araştırmacilar ve alan uzmanları, ebeveynler, çocuklar, personel/çalışanlar olarak dört farklı bakış açısıyla ele alınmaktadır. Ortamın özellikleri, materyallerin durumu yetişkinlerin bakış açısıyla yansıtılarak; sınıfın çocuklara nasıl deneyimler sunduğu değerlendirilmeye çalışılarak; okula dışarıdan bir bakış açısı oluşturan ailelerin aldığ hizmetler ele alınarak ve kaynaştırma programlarındaki personelin deneyimleri de ortaya konularak kalite değerlendirilebilmektedir (Katz, 1993). Bununla birlikte, Loreman ve diğ. (2014) kaynaştırma uygulamalarının daha geniş bir kapsamda mikro, mezo ve makro düzeylerde göstergelerle değerlendirilebileceğini belirtmiştir. Mikro düzey, sınıfları ve bireyleri; mezo düzey okulları ve makro düzey ise tüm bu düzeylerin bulunduğu sistemi ifade etmektedir. Mikro düzeyde öğretmenler, okul öncesi kaynaştırma ortamlarının kalitesine ilişkin kritik rol üstlenmekte ve kaliteye etki eden önemli faktörlerden biri olarak karşımıza çıkmaktadır. Makro düzeyde ise kaynaştırmaya yönelik tutumlar, politikalar, paylaşılan ortak bir vizyonun oluşması, eğitim ve destek hizmetler; sınıf, okul ve organizasyon yapıları ve toplum, okul öncesi kaynaştırmayı ve kalitesini etkileyen anahtar yapılar olarak görülmektedir (Buysse, Wesley, Bryant ve Gardner, 1999; Lieber ve diğ., 2000; Loreman, 2007). Bu bağlamda okul öncesi kaynaştırmanın kalitesi; öğretmenlerin ve öğrencilerin özellikleri, programın değişkenleri, ögrretimsel stratejiler, aileler ve uzmanlar arasındaki işbirliği, fiziksel ortamın düzenlenmesi ve materyalleri, günlük rutinleri uyarlama ve bireyselleştirme gibi pek çok değişkenle birlikte ele alınabilmektedir (Cate, Diefendorf, McCullough, Peters ve Whaley, 2010).

Okul öncesi kaynaştırmanın kalitesi kapsamında ele alınabilecek birçok özellik bulunmaktadır. Bu özellikler sınıfta bulunan öğretmen-çocuk oranı, grup büyüklüğü, öğretmenin eğitim durumu gibi değişkenleri içeren yapısal kalite (structural quality), öğretmen-çocuk etkileşimi, eğitim programının uygulanması gibi çocukların günlük deneyimlerine doğrudan etki eden süreç kalitesi (process quality) ve iki boyutun birleşiminden oluşan genel kalite (global quality veya overall quality) boyutlarında ele alınmaktadır (Hestenes, Cassidy, Hedge ve Lower, 2007; Hestenes, Cassidy, Shim ve Hedge, 2008; Phillipsen, Burchinal, Hower ve Cryer, 1997). Yapisal kalite daha çok düzenlenebilir özellikleri içerirken, süreç kalitesi çevresel özellikleri içermektedir (Wolery ve Odom, 2000). Yapısal kalitenin ölçülmesi görece daha kolaydır ve görüşmelerle bilgi alınabilmektedir. Diğer taraftan süreç kalitesi belli bir zaman diliminde farklı ortamlarda ayrıntılı, uzun süreli ve kapsamlı gözlemler gerektirmektedir (Ishimine ve Tayler, 2014). Genel kalite ise; süreç kalitesi ve yapısal kalite ile birlikte kalitenin pek çok boyutu kapsayacak biçimde bütüncül olarak ele alınmasını gerektirmektedir. Yapısal, süreç ve genel kalite özellikleri daha çok araştırmacılar ve profesyonellerin kaliteye bakış açılarını yansıtmaktadır.

Genel kalitenin değerlendirilmesi, The Division for Early Childhood of the Council for Exceptional Children (DEC) ve The National Association for the Education of Young Children (NAEYC) kuruluşlarının 2009 yılında ortak olarak yayınladıkları belgede belirttikleri erişim (access), katılım (participation) ve destek (support) boyutlarındaki bileşenlere yönelik değerlendirmenin sağlanması, çok boyutlu veri toplama ile birlikte genel kalite göstergelerinin ortaya konulmasını ve değerlendirilmesini gerektirmektedir (DEC ve NAEYC, 2009). Erişim, fiziksel engelleri kaldırarak çocuklara çok çeşitli etkinlikler sunmak ve ortam sağlamak, onların en iyi şekilde gelişmelerini ve öğrenmelerini desteklemek için gerekli uyarlamaları yapmak olarak ele alınmaktadır. Evrensel tasarım ilkelerinin hem fiziksel ortamda hem de öğretim ortamında ele alınarak işler hale getirilmesi de erişim boyutunda vurgulanmaktadır (Barton ve Smith, 2015). Katılım ise, erişim boyutunun ötesinde çocuklarda aidiyet hissi oluşturarak oyun ve öğrenme etkinlikleriyle meşgul olmalarını sağlamak şeklinde ele alınmakta ve destek, yüksek kaliteli kaynaştırma uygulamaları için sistemlerin alt yapısını oluşturmak şeklinde ifade edilmektedir. Bununla birlikte, kaynaştırmanın, her çocuğun ve ailesinin haklarını destekleyen değerleri, politikaları ve uygulamaları kapsadı̆̆ı, yetersizlikleri gözetilmeksizin her çocuğun toplumun bir üyesi olarak çeşitli etkinliklere 
katılımını içerdiği, tüm bu boyutların okul öncesi dönemde kaliteli kaynaştırma uygulamalarının temelini oluşturduğu belirtilmektedir (DEC ve NAEYC, 2009).

Okul öncesi kaynaştırmanın kalitesine ilişkin alanyazında vurgulanan bir diğer nokta da okul öncesi eğitimin, kaynaştırmanın kalitesini de etkilemesidir. "Okul öncesi kaynaştırma”nın kalitesinin değerlendirilmesi kapsamını oluşturan alt yapı “okul öncesi eğitim ortamlarının kalitesi”ne yönelik çalışmalardan oluşmakta, 2000'li yılların sonundan itibaren okul öncesi kaynaştırmanın kalitesi özelinde çabalar olduğu görülmektedir. Yüksek kaliteli okul öncesi eğitim programları, okul öncesi kaynaştırma programlarının kalitesi için gereklidir ancak yeterli değildir. Kaynaştırma ortamında yetersizliği olan çocukların gelişimsel gereksinimlerinin de karşılanması ön plana çıkmaktadır (Wolery ve Odom, 2000). Türkiye'de okul öncesi eğitimin kalitesi ile ilgili yapılan araştırmaların sınırlı olduğu görülmekte (Yılmaz, 2014), kaynaştırmanın kalitesinin son yıllarda elealınmaya başlandığı dikkati çekmektedir. Milli Eğitim Bakanlığı'nın (MEB) okul öncesi eğitimin kalitesine ve standartlarını geliştirmeye yönelik girişimlerinin olduğu, okul öncesi dönemde özel gereksinimli çocukların eğitim ve gelişim haklarına duyarlı, tüm çocukların gelişimini destekleyen standartların oluşturulmaya başlandığı görülmektedir (MEB, 2015).

\section{Okul Öncesi Kaynaştırmanın Kalitesinin Değerlendirilmesi}

Kalite değerlendirme, genellikle belli yaklaşımlar çerçevesinde düzenleme/denetleme, araştırmalar kapsamında politika geliştirmeye katkı sağlama ve uygulamaların geliştirilmesi amacıyla yapılmaktadır (SirajBlatchford ve Wong, 1999). Uluslararası alanyazında özel gereksinimli çocukların kaynaştırma ortamlarından en üst düzeyde faydalanabilmesi, buna ilişkin modeller geliştirilmesi ve bunun için var olan durumun ortaya konulması amacıyla kaynaştırma ortamlarının kalitesini değerlendirmeye yönelik araştırmalar bulunmaktadır (Fyssa ve Vlachou, 2015; Hestenes ve diğ., 2008; La Paro, Sexton ve Snyder, 1998). Okul öncesi kaynaştırmanın kalitesinin değerlendirilmesi amacıyla kalite kavramına farklı yaklaşımları temel alan araçların geliştirildiği görülmektedir (Cate ve diğ., 2010). Bu araçların geliştirilme gereksinimi farklı nedenlerle ortaya çıkmaktadır:

1. Kanıt temelli uygulamalar ve politikalara katkı sağlamak üzere çocukların kaynaştırma programlarındaki deneyimlerinin uzun ve kısa vadedeki etkilerini değerlendirme,

2. Programlara alternatif maddi kaynak sağlamaya ve desteklemeye yönelik değerlendirme ile profesyonel gelişimi sağlama,

3. Programların etkililiğini geliştirmeye yönelik öz değerlendirme sunma,

4. Kaynaştırmanın kalite standartlarını geliştirme,

5. Hesap verebilirlik ve politikaları değerlendirme amacıyla okul öncesi kaynaştırmanın kalitesini değerlendirmeye yönelik çeşitli araçların geliştirilmesine gereksinim duyulmaktadır (Lero, 2010).

$\mathrm{Bu}$ bağlamda, okul öncesi kaynaştırmanın kalitesini değerlendirmeye yönelik araştırmalarda yaygın olarak kullanılan araçlar ve özellikleri aşağıda belirtilmiştir:

Okul Öncesi Eğitim Ortamı Değerlendirme Ölçeği (Early Childhood Environment Rating ScaleECERS). Harms, Cryer ve Clifford tarafindan 1980 yılında geliştirilen ve 2007 yılında gözden geçirilen (ECERSRevised) ölçek, 2,5-5 yaş aralığında bulunan çocuklara uygulanan okul öncesi eğitim programının ve ortamının özelliklerini değerlendirmek için kullanılmaktadır (Cate ve diğ., 2010). Sınıf alanı ve mobilyalar, kişisel bakım rutinleri, dil ve akıl yürütme, etkileşim, etkinlikler, program yapısı, aile ve personel olmak üzere yedi alt boyut ve 43 madde içermektedir. Bu araç, erken çocukluk eğitimi programlarının boyutlarını geçerli ve yararlı biçimde değerlendirse de özellikle sınıf ortamındaki uygulamaları ve yetersizliği olan çocukları kapsayacak şekilde geliştirilmemiştir (Soukakou, 2012). Ancak bazı araştırmalarda kaynaştırma ortamını değerlendirmeye yönelik olarak kullanılmıştır (Buysse ve diğ., 1999; Hestenes ve diğ., 2008; La Paro ve diğ., 1998). ECERS'ın 2015 yılında güncellenen son sürümü altı alt boyutta yer alan 35 madde içermekte, 3-5 yaş çocuklarının bulunduğu okul öncesi eğitim ortamlarının kalitesini değerlendirmek amacıyla kullanılmaktadır (http://www.ersi.info/ecers3.html). 
Çeşitli dillere çevrilen ve yaygın olarak kullanılan bu ölçek, ülkemizde erken çocukluk eğitim ortamlarının kalitesini değerlendirmeye yönelik araştırmalarda da kullanılmıştır (Aksoy, 2009; Feyman, 2006; Göl-Güven, 2009; Güçhan-Özgül, 2011; Kalkan, 2008; Solak, 2007; Tekmen, 2005). Bazı sınırlılıkları olmasına karşın konuya ilişkin yararlı bir değerlendirme aracı olarak görülmektedir (Ishimine ve Tayler, 2014). Ölçeğin, 0-30 aylık kü̧̈ük çocukların bulunduğu ortamların değerlendirilmesine yönelik versiyonu da bulunmaktadır. "Infant/Toddler Environment Rating Scale" adı verilen ölçekte sınıf alanı ve mobilyalar, kişisel bakım ve rutinler, dinleme ve konuşma, etkinlikler, etkileşim, program yapısı, aile ve personel olmak üzere yedi alt boyut ve 39 madde yer almaktadır. Aracın kullanımı uzmanlar tarafindan verilen eğitimi gerektirmektedir.

Kaynaştırma Deneyimlerinin Kalitesi Ölçeği (The Quality of Inclusive Experiences MeasureQuIEM). Wolery, Pauca, Brashers ve Grant tarafından kaynaştırma ortamında bulunan özel gereksinimli çocukların deneyimlerini değerlendirmek üzere 2000 yılında geliştirilmiştir (akt. Soukakou 2015). Eğitim ortamında uygulanan programın amaçları, kurum çalışanlarının desteği, fiziksel çevrenin yeterliği ve erişilebilirliği, katılım, yetişkin-çocuk iletişimi ve etkileşimi, bireyselleştirme ve çocuk-çocuk iletişimi ve etkileşimi olarak yedi boyutta ele alınan bir kapsama sahiptir. Ölçek bu kapsamı ile kaynaştırma eğitimi alan çocuğun deneyimlerinin zaman örneklemi alınarak ve olay kaydı yapılarak gözlenmesi, kurumda çalışanlarla görüşme yapılması ve dokümanların gözden geçirilmesi ile kaynaştırmanın genel kalitesine ilişkin bilgi vermektedir. QuIEM ile kaynaştırma programlarının kalitesinin ölçülebilmesi için, kullanımını geçerli hale getirecek daha fazla araştırma sonucuna gereksinim duyulduğu vurgulanmaktadır (Odom ve diğ., 2011). Ölçeğin geçerlik ve güvenirlik kapsamında psikometrik özelliklerine ilişkin daha fazla çalışması yapılması gerektiğini belirtilmektedir (Fyssa ve Vlachou, 2015; Spiker, Hebbeler ve Barton, 2011).

SpeciaLink Erken Çocuklukta Kaynaştırmanın Kalitesini Derecelendirme Ölçeği (The SpeciaLink Early Childhood Inclusion Quality Rating Scale-SECIQR). Irwin tarafindan 2005 yılında geliştirilen "SpeciaLink Erken Çocuklukta Kaynaştırma Uygulamaları ve İlkeleri Ölçeği (SpeciaLink Child Care Inclusion Practices Profile and Principles Scale)" temelinde ortaya konulan ve 2009 yllında son hali verilen ölçek (akt. Lero, 2010; Soukakou, 2012) uygulamalar ve ilkeler olarak iki bölümden oluşmaktadır (http://www.specialinkcanada.org/about/history.html). Fiziksel ortam, kurum çalışanlarının eğitimi, terapiler, okula geçiş sistemi hazırlığı, materyaller vb. gibi 11 madde ve 158 göstergenin yer aldığı uygulama bölümü; tam katılım, sıfir ayrıştırma, liderlik, önleyici stratejiler vb. gibi altı madde ve 92 göstergenin yer aldığı ilkeler bölümü bulunmaktadır. Kaynaştırma kalitesi gözlem, doküman incelemesi ve görüşmeler yoluyla veri toplanarak incelenmektedir (Lero, 2010). Bu ölçek, kaynaştırma uygulamalarının çocuklar üzerindeki kısa ve uzun vadedeki etkilerini değerlendirerek, kanıt temelli politika ve uygulamalara katkı sağlamakta; programları değerlendirerek profesyonel gelişime destek olabilecek modeller geliştirme amacıyla kullanılabilecek kalite standartlarının geliştirilmesi için bilgi sağlamaktadır (Cate ve diğ., 2010). ECERS gibi her madde yedili dereceleme ile puanlanmakta; 17 maddede 250 özel gösterge kapsamında değerlendirme yapılmaktadır (Spiker ve diğ.,2011). Ölçekle toplanan veriler okul öncesi kaynaştırma ortamlarının genel kalitesinden çok yapı kalitesine ilişkin bilgi vermektedir. Bu ölçeğin psikometrik özelliklerinin daha çok araştırma ile desteklenmeye ihtiyacı olduğu belirtilmiştir (Soukakou, 2012).

Kaynaştırma Sınıf Profili-KSP (Inclusive Classroom Profile-ICP). Bu araç, okul öncesi kaynaştırma uygulamaları yürütülen, 2-5 yaş çocuklarının olduğu ve gelişimsel yetersizliğinden dolayı tanı alan en az bir çocuğun bulunduğu sınıflarda uygulanmaktadır. Ölçeğin İngiltere'de pilot uygulaması yapılarak geçerlik ve güvenirliğinin kabul edilebilir düzeyde olduğu belirlenmiş ve sonrasında farklı çalışmalarla da psikometrik özellikleri test edilmiştir (Soukakou, 2012; Soukakou, Winton ve West, 2012; Soukakou ve diğ., 2015). KSP'de ortam ve materyallerin uyarlanması, yetişkinin akran etkileşimine katılımı, çocukların oyununa yetişkin rehberliği, çatı̧̧ma çözme, katılım, yetişkin-çocuk sosyal etkileşimi, sosyal iletişim için destekleme, grup etkinliklerini uyarlama, etkinlikler arası geçiş, geribildirim, çocukların bireysel gereksinimlerini ve amaçlarını planlama ve izleme olmak üzere 12 madde bulunmaktadır. Her bir maddenin operasyonel tanımı yapılmış ve puanlamasına iliş̧kin özellikler ayrıca açıklanmıştır. KSP yedi dereceli olarak puanlanan likert tipi ölçektir, uygun olmayan ve 
hatta zararı olabilecek uygulamalara bir puan, bireyselleştirmeyi desteklerken kaynaştırmaya katkı sağlayabileceği düşünülen uygulamalara ise yedi puan verilmektedir. İngiltere'de geliştirilmiş ve Amerika Birleşik Devletleri'nde (ABD) de kullanılan KSP, diğer ülkelere de uyarlanabilecek şekilde düzenlenmiştir (Soukakou, 2012). DEC ve NAEYC kuruluşlarının kaliteli kaynaştırma uygulamaları kapsamındaki önerileri doğrultusunda, okul öncesi kaynaştırma ortamlarının genel kalitesine yönelik bilgi vermenin yanı sıra profesyonel gelişim, programı değerlendirme ve politika geliştirme amacı ile de kullanılabilmektedir (Soukakou ve diğ., 2015). Kullanımı, standart bir eğitim süreci ve sertifika sahibi olmayı gerektirmektedir. Araç ilk defa Yılmaz (2014) tarafindan Türkçe'ye çevrilmiş ve Ankara' da bulunan bağımsız anaokulları ve özel okul öncesi kurumlarındaki kaynaştırma sınıflarının kalitesi belirlenmeye çalışılmıştır.

Kalite Değerlendirme ve Geliştirme Sistemi (Quality Rating and Improvement System-QRIS). ABD'de pek çok eyalette erken çocukluk eğitimi programlarının kalitesini değerlendirmek, ailelerin yüksek kaliteli erken çocukluk eğitimi konusundaki taleplerini artırmak ve eğitim hizmeti sunanlarda profesyonel gelişimi sağlamak üzere ortaya konulmuş bir sistemdir (Cate ve diğ., 2010). Bu sistem birçok eyalet programının kalitesini değerlendirmek, programlarının kalitesinin gelişimlerini izlemek ve bununla ilgili bilgileri aileler ve diğer paydaşlara sunmak üzere kullanılmaktadır. Bazı eyaletlerin kullandıkları QRIS sisteminde yetersizliği olan çocuklar ve ailelerine yönelik performans standartları bulunmaktadır. $\mathrm{Bu}$ standartlar incelendiğinde kaynaştırmanın genel program kalitesini ele almaya yönelik bir görüş birliği bulunmasa da kaynaştırmanın farklı boyutlarının standartlar temel alınarak vurgulandığı (Örn; uyarlamalar, bireyselleştirilmiş hizmet planlarındaki amaçlara yönelik etkinlikler, profesyonel gelişim vb.) görülmektedir (National Professional Development Center on Inclusion [NPDCI], 2009). QRIS, ABD'de erken çocukluk eğitim programları ve okul öncesi kaynaştırmaya ilişkin kaliteli programlar oluşturmaya yönelik standartların ortaya konulmasında etkili olarak kullanılmaktadır.

Özetle, psikometrik özellikleri bakımından güçlü ve zayıf yanları olduğu ifade edilen araçların gözlem, görüşme ve doküman incelemesi yolu ile çok boyutlu olarak özellikle genel kaliteyi değerlendirmeye yönelik veri sağladıkları dikkati çekmektedir. ECERS okul öncesi ortamlarını değerlendirmede en yaygın kullanılan ölçek olmakla birlikte, son güncellemeleri ile okul öncesi kaynaştırma ortamlarının değerlendirilmesine yönelik de kullanıldığı ifade edilmektedir. QuIEM diğer araçlara göre sınırlı veri sağlamakta, araştırmalarca desteklenen bulgularının ortaya konulması gerekmektedir. SECIQR daha çok yapı kalitesine ilişkin bilgi vermekte, diğer araçlardan maddeleri kategorize etme bakımından farklılaşmaktadır. $K S P$ yeni/güncel bir ölçek olması nedeniyle, okul öncesi kaynaştırmanın kalitesi boyutunda oluşan alanyazını DEC ve NAEYC kriterleri doğrultusunda dikkate alması ile daha kapsamlı bir bakış açısı sunmakta ve sadece kalite değerlendirmeye yönelik değil profesyonel gelişim, izleme ve politika geliştirmeye yönelik de kullanılabilmektedir. Kalite değerlendirmeye yönelik kullanılan araçların, programların hem kalitesini değerlendirmek, hem gelişimini izlemek, hem de profesyonel gelişim standartlarının oluşumuna katkı sağlayarak bölge bazında uygulanmasına örnek oluşturması QRIS sistemi ile görülmektedir. Bununla birlikte, özel olarak okul öncesi kaynaştırmanın kalitesini değerlendirmeye yönelik bir sistem olmadığı için $Q R I S^{\prime}$ in yaygın etki alanıyla birlikte sınırlı veri sağladığı söylenebilmektedir.

\section{Sonuç ve Öneriler}

Bu makale ile bir sonuca ulaşmaktan çok son yıllarda uluslararası alanyazında sıklıkla ele alınan "okul öncesi kaynaştırmanın kalitesi”, kalite değerlendirmeyle ilgili boyutları ve bu doğrultuda kullanılabilecek değerlendirme araçları konusunda başlangıç oluşturabilecek bilgilerin gündeme getirilmesi amaçlanmıştır. Okul öncesi kaynaştırma, erken çocukluk döneminde gelişimsel yetersizliği olan çocuklara ve ailelerine yönelik önemli hizmetlerin belli bir sistematik içerisinde sunulmasını gerektiren ve gerek özel gereksinimli çocuklara gerekse normal gelişim gösteren çocuklara farklı boyutlarda yarar sağlayan bir alandır (Henninger ve Gupta, 2014). Bu alanda sunulan hizmetlerin niteliğinin artması ve yüksek kaliteli kaynaştırma uygulamalarının ortaya konulması öncelikle "kalite"nin hangi boyutları ve hangi özellikleri kapsadığının ortaya konulmasını gerektirmektedir. Gelişimsel yetersizliği bulunan öğrencilerin, normal gelişim gösteren çocuklarla aynı ortamda bulunmasının ötesinde kaynaştırma uygulamalarını nitelikli hale getiren kaliteli uygulamaların özellikleridir. Okul öncesi kaynaştırmada kalite, yetersizlikten etkilenen çocukların bulunduğu sınıfın özelliklerinden, sınıftaki etkileşim 
ortamına; öğretmenlerin deneyimleri ve aldıkları ücretten ebeveynlerle işbirliğine kadar yapısal ve süreçsel pek çok özelliği kapsamakta, bu özellikleri farklı biçimlerle ele alan ölçme araçları bulunmaktadır. Bu araçların profesyonelce geliştirilmesi ve araştırmalarda kullanılması, yüksek kaliteli okul öncesi kaynaştırma uygulamaları için anahtar olarak görülmektedir. Bununla birlikte, okul öncesi kaynaştırmanın erişim boyutunda, öğrenme için evrensel tasarım ilkeleri ve destekleyici teknolojilerin kullanılması; katılım boyutunda, gömülü öğretim, doğal öğretim yöntemleri ve diğer destekleyici stratejilere sınıf uygulamalarında yer verilmesi; destek boyutunda ise profesyonel gelişim, işbirliği modelleri, aile-uzman işbirliğine yönelik çalışmaların yapılması önerilmektedir (NPDCI, 2011). Türkiye'deki kaynaştırma uygulamaları bağlamında erişim, katılım ve destek boyutunda çalışmaların yapılmasının okul öncesi kaynaştırmanın "nasıl" etkili hale gelebileceğine ilişkin önemli veriler sağlayacağı düşünülmektedir. Bununla birlikte; "okul öncesi kaynaştırmanın kalitesi" ile ilgili gelişmelerin, "okul öncesi eğitimin kalitesi" ile ilgili olduğu göz önünde bulundurulduğunda, bu kapsamda ülkemizde her iki alan odağında da çok daha fazla araştırmaya gereksinim olduğu düşünülmektedir. Ayrıca, araştırmalarda süreç kalitesi, yapısal kalite ve genel kalite özelliklerine ilişkin boyutların, okul öncesi kaynaştırmanın kalitesi ile ilişkisinin ortaya konulmasına yönelik çalışmalar yapılmasının önemli olduğu görülmektedir. Yüksek kaliteli okul öncesi kaynaştırma ortamlarının önündeki engellerin personel eğitimi, okul öncesi eğitim ve özel eğitimciler arasındaki işbirliği ile ilişkili olduğu belirtilmektedir (Odom ve Bailey, 2001). Bu noktadan hareketle, öğretmenlerin profesyonel gelişimi ile ilgili yapılacak farklı çalışmaların okul öncesi kaynaştırmanın kalitesine etkisini inceleyen araştırmalara gereksinim olduğu dikkat çekmektedir. Yüksek kaliteli kaynaştırma uygulamalarının ortaya konulması kadar, bu uygulamaların sisteme entegre edilmesi ve geniş çapta ele alınıp yaygınlaştııılması da önemlidir. Bu noktada, kalite standartlarının oluşturulması için değerlendirme araçlarının sunduğu bilgilerle oluşturulacak veri temelli sistemin okul öncesinde kaynaştırma ortamlarında bulunan çocuklar, öğretmenleri ve aileleri için anlamlı ve yararlı olabileceği düşünülmektedir. 
Kaynaklar

Adams, D. (1993). Defining educational quality. Improving Educational Quality Project Publication, 1. Retrieved from http://pdf.usaid.gov/pdf_docs/PNACA245.pdf.

Aksoy, P. (2009). Okul öncesi eğitim kurumlarının eğitim ortamlarının niteliğinin bazı değişkenler açısından incelenmesi (Tokat ili örneği) [Analysis of the quality of preschool inclusion clasrooms in terms of some variables (Example of the Tokat province)] (Unpublished master's thesis) Ankara Üniversity, Institute of Educational Sciences, Ankara, Turkey). Retrieved from: http://acikarsiv.ankara.edu.tr/browse/4404/

Barton, E. E., \& Smith, B. J. (2014). Fact sheet of research on preschool inclusion. Pyramid Plus: The Colorado Center for Social Emotional Competence and Inclusion. Denver, CO. Retrieved from http://www.pyramidplus.org/.

Barton, E. E., \& Smith, B. J. (2015). Advancing high-quality preschool inclusion: A discussion and recommendations for the field. Topics in Early Childhood Special Education, 35(2), 69-78.

Batu, S., \& Kırcaali-İftar, G. (2005). Kaynaştırma [Inclusion]. Ankara: Kök Yayınları.

Bruder, M. B. (2010). Early childhood intervention: A promise to children and families for their future. Exceptional Children, 76(3), 339-355.

Bryant, D. M., Burchinal, M., Lau, L. B., \& Sparling, J. J. (1994). Family and classroom correlates of head start children's developmental outcomes. Early Childhood Research Quarterly, 9(3-4), 289-309.

Burchinal, M. R., Roberts, J. E., Nabors, L. A., \& Bryant, D. M. (1996). Quality of center child care and infant cognitive and language development. Child Development, 67(2), 606-620.

Burchinal, R. M., Roberts, J. E., Riggings, R., Zeisel, S. A., Neebe, E., \& Bryant, D. (2000). Relating quality of center-based child care to early cognitive and language development longitudinally. Child Development, 2(71), 339-357.

Buysse, V., \& Bailey, D. B. (1993). Behavioral and developmental outcomes in young children with disabilities in integrated and segregated settings: a review of comparative studies. The Journal of Special Education, 26(4), 434-461.

Buysse, V., \& Hollingsworth, H. (2009). Program quality and early childhood inclusion: Recommendations for professional development. Topics in Early Childhood Special Education, 2(29), 119-128.

Buysse, V., Wesley, P. W., Bryant, D., \& Gardner, D. (1999). Quality of early childhood programs in inclusive and noninclusive settings. Exceptional Children, 65(3), 301-314.

Cate, D., Diendorf, M., McCullough, K., Peters, M. L., \& Whaley, K. (Eds.). (2010). Quality indicators of inclusive early childhood programs/practices: A compilation of selected resources. Chapell Hill: The University of North Carolina, FPG Child Development Institute, National Early Childhood Technical Center. Retrieved from http://www.nectac.org/ pdfs/pubs/qualityindicatorsinclusion.pdf

The Division for Early Childhood of the Council for Exceptional Children (DEC) andThe National Association for the Education of Young Children (NAEYC) (2009). Early childhood inclusion: A joint position statement of the Divisionfor Early Childhood (DEC) and the National Association for the Education of Young Children (NAEYC). Chapel Hill, NC: The University of North Carolina, FPG Child Development Institute. $\quad$ Retrieved https://www.naeyc.org/files/naeyc/file/positions/DEC_NAEYC_EC_updatedKS.pdf

Feyman, N. (2006). Okul öncesi eğitim kurumlarında kalitenin çocukların gelişim alanları üzerine etkisinin incelenmesi [Examination of the effect of the quality of kindergartens on the developmental areas of 
children] (Unpublished master's thesis, Hacettepe University, Institute of Social Sciences, Ankara, Turkey). Retrieved from: https://tez.yok.gov.tr/UlusalTezMerkezi/tezSorguSonucYeni.jsp

Fyssa, A., \& Vlachou, A. (2015). Assessment of quality for inclusive programs in Greek preschool classrooms. Journal of Early Intervention, 37(3), 190-207.

Green, K., Terry, N., \& Gallagher, P. (2014). Progress in language and literacy skills among children with disabilities in inclusive early reading first classrooms. Topics in Early Childhood Special Education, 33(4), 249-259.

Göl-Güven, M. (2009). Evaluation of the early childhood classrooms in Turkey. Early Childhood Development and Care, 179(4), 437-451.

Güçhan-Özgül, S. (2011). Okul öncesi eğitim ortamlarının kalite değişkenleri açısından değerlendirilmesi [Evaluating the preschool inclusion settings in terms of quality variables] (Unpublished master's thesis), Bakıkesir University, Institute of Social Sciences, Balıkesir.

Henninger IV, W. R., \& Gupta, S. S. (2014). How do children benefit from inclusion? In W. R. Henninger IV, S. S. Gupta \& M. E. Vinh (Eds), First steps to preschool inclusion: How to jumpstart your program wide plan. (pp 33-57) Retrieved from http://products.brookespublishing.com/First-Steps-to-PreschoolInclusion-P762.aspx

Hestenes, L. L., Cassidy, D. J., Hedge, A. V., \& Lower, J. K. (2007). Quality in inclusive and noninclusive infant and toddler classrooms. Journal of Research in Childhood Education, 22(1), 69-84.

Hestenes, L. L., Cassidy, D. J., Shim, J., \& Hedge, A. V. (2008). Quality in inclusive preschool classrooms. Early Education and Development, 19(4),519-540.

Hollingsworth, H. L., \& Buysse, V. (2009). Establishing friendships in early childhood inclusive settings: What roles do parents and teachers play? Journal of Early Intervention, 31(4), 287-307.

Hunt, P., Farron-Davis, F., Beckstead, S., Curtis, D., \& Goetz, L. (1994). Evaluating the effects of placement of students with severe disabilities in general education versus special classes. Journal of the Association for Person with Severe Handicaps, 19(3), 200-214.

Ishimine, K., \& Tayler, C. (2014). Assessing quality in early childhood education and care. European Journal of Education, 49(2), 272-290.

Kalkan, E. (2008). Okul öncesi eğitim kurumlarında kalitenin fiziksel koşullar açısından incelenmesi [Assessing quality in preschool classrooms in terms of physical conditions] (Unpublished master's thesis, Hacettepe University, Institute of Social Sciences, Ankara, Turkey). Retrieved from: https://tez.yok.gov.tr/UlusalTezMerkezi/tezSorguSonucYeni.jsp

Kargın, T. (2004). Kaynaştırma: Tanımı, gelişimi ve ilkeleri [Inclusion: definition, development and principles] Ankara Üniversitesi Ĕ̆itim Bilimleri Fakültesi Özel Eğitim Dergisi, 5(2),1-13.

Katz, L. G. (1993). Multiple perspectives on the quality of early childhood programmes. European Early Childhood Education Research Journal, 1(2), 5-9.

La Paro, K. M., Sexton, D., \& Snyder, P. (1998). Program quality characteristics in segregated and inclusive early childhood settings. Early Childhood Research Quarterly,13(1),151-167.

Layzer, J. I., \& Goodson, B. D. (2006). The "quality" of early care and education settings definitional and measurement issues. Evaluation Review, 30(5), 556-576. 
Lero, D. S. (2010). Assessing inclusion quality in early learning and child care in Canada with the SpeciaLink Child Care Inclusion Practices Profile and Principles Scale. SpeciaLink National Centre for Child Care Inclusion. Retrieved from http://www.specialinkcanada.org/about/pdf/SpeciaLink\%20Research\%20Report\%20on\%20Inclusion\% 20Quality\%20Rating\%20Scale.pdf.

Lieber, J., Hanson, M. J., Beckman, P. J., Odom, S. L., Sandall, S. R., Schwartz, I. S., Horn, E., \& Wolery, R. (2000). Key influences on the initiation and implementation of inclusive preschool programs. Exceptional Children, 67(1), 83-98.

Lipsky, D. K., \& Gartner, A. (1996). Inclusion, school restructuring and the remaking of American society. Harvard Educational Review, 66(4), 762-796.

Loreman, T. (2007). Seven pillars of support for inclusive education: moving from "why?" to" how?". International Journal of Whole Schooling, 3(2), 22-38.

Loreman, T., Forlin, C., \& Sharma, U. (2014). Measuring indicators of inclusive education: A systematic review of the literature. In C. Forlin \& T. Loreman (Eds.) Measuring inclusive education (International perspectives on inclusive education) (pp.165-187). Emerald Group Publishing.

Milli Eğitim Bakanlığı (2015). Okul öncesi eğitim ve ilköğretim kurumları standartları kılavuz kitabı [Guidance for preschool education and primary school standards]. Retrieved from https://tegm.meb.gov.tr/meb_iys_dosyalar/2015_04/09112933_kurumstandartlarklavuzkitap.pdf.

National Professional Development Center on Inclusion (2009). Research synthesis points on early childhood inclusion. Chapel Hill: The University of North Carolina, FPG Child Development Institute. Retrieved from http://npdci.fpg.unc.edu/sites/npdci.fpg.unc.edu/files/resources/NPDCI-ResearchSynthesisPoints10-2009_0.pdf.

National Professional Development Center on Inclusion (2011). Research synthesis points on practices that support inclusion. Chapel Hill: The University of North Carolina, FPG Child Development Institute. Retrieved from http://npdci.fpg.unc.edu/sites/npdci.fpg.unc.edu/files/resources/NPDCIResearchSynthesisPointsInclusivePractices-2011_0.pdf.

Odom, S. L., \& Bailey, D. (2001). Inclusive preschool programs: Classroom ecology and child outcomes. In M. J. Guralnick (Ed.), Early childhood inclusion: Focus on change (pp. 253-276). Baltimore: Brookes Publishing.

Odom, S. L., Hanson, M. J., Lieber, J., Marquart, J., Sandall, S., Wolery, et al. (2001). The costs of preschool inclusion. Topics in Early Childhood Special Education, 21(1), 46-55.

Odom, S. L., Buysse, V., \& Soukakou, E. (2011). Inclusion for young children with disabilities: A quarter century of research perspectives. Journal of Early Intervention, 4, 344-356.

Odom, S. L., Zercher, C., Li, S., Marquart, J. M., Sandall, S., \& Brown, W. H. (2006). Social acceptance and rejection of preschool children with disabilities: A mixed-method analysis. Journal of Educational Psychology, 98(4), 807-823.

Phillipsen, L. C., Burchinal, M. R., Howes, C., \& Cryer, D. (1997). The prediction of process quality from structural features of child care. Early Childhood Research Quarterly, 12(3), 281-303.

Rafferty, Y., Piscitelli, V., \& Boettcher, C. (2003). The impact of inclusion on language development and social competence among preschoolers with disabilities. Exceptional Children, 69(4), 467-479.

Salend, S. J. (1998). Effective mainstreaming creating inclusiveclassroom. Ohio: Merrill. 
Siraj-Blatchford, I., \& Wong, Y. L. (1999). Defining and evaluating 'quality' early childhood education in an international context: Dilemmas and possibilities. Early Years, 20(1), 7-18.

Solak, N. (2007). Adana il merkezinde bulunan okul öncesi eğitim kurumlarında kalitenin incelenmesi [Determining of quality level of the government preschools and private schools in Adana province] (Unpublished master's thesis, Çukurova University, Institute of Social Sciences, Adana, Turkey). Retrieved from: http://library.cu.edu.tr/tezler/6477.pdf

Soukakou, E. (2012). Measuring quality in inclusive preschool classrooms: Development and validation of the Inclusive Classroom Profile (ICP). Early Childhood Research Quarterly, 27(3), 478-488.

Soukakou, E., Winton, P., \& West, T. (2012). The inclusive classroom profile (ICP): Report on preliminary findings of demonstration study in North Carolina. Chapell Hill, NC: NPDC, FPG Child Institute.

Soukakou, E. P., Winton, P. J., West, T. A., Sideris, J. H., \& Rucker, L. M. (2015). Measuring the quality of inclusive practices findings from the inclusive classroom profile pilot. Journal of Early Intervention, $36(3), 223-240$.

Spiker, D., Hebbeler, K. M., \& Barton, L. R. (2011). Measuring quality of ECE programs for children with disabilities. In M. Zaslow, I. Martinez-Beck, K. Tout \& T. Halle (Eds.), Quality measurement in early childhood settings (pp. 229-256). Baltimore, MD: Paul H. Brookes Publishing.

Tekmen, B. (2005). A study on the structural and process of early childhood and care centers in Ankara. (Unpublished master's thesis), Middle East Technical University, The Graduate School of Social Sciences, Ankara.

Wolery, R. A., \& Odom, S. (2000). An administrator's guide to preschool inclusion. Chapel Hill: University of North Carolina, FPG Child Development Center, Early Childhood Research Institute on Inclusion. Retrieved from http://fpg.unc.edu/node/784

Vlachou, A., \& Fyssa, A. (2016). 'Inclusion in practice': Programme practices in mainstream preschool classrooms and associations with context and teacher characteristics. International Journal of Disability, Development and Education, 63(5), 529-544.

Yılmaz, B. (2014). Okul öncesi kaynaştırma sınıflarının kalitesinin değerlendirilmesi [Evaluating preschool inclusion classrooms' quality] (Unpublished master's thesis, Gazi University, Institute of Educational Sciences, Ankara, $\quad$ Turkey). $\quad$ Retrieved from https://tez.yok.gov.tr/UlusalTezMerkezi/tezSorguSonucYeni.jsp 


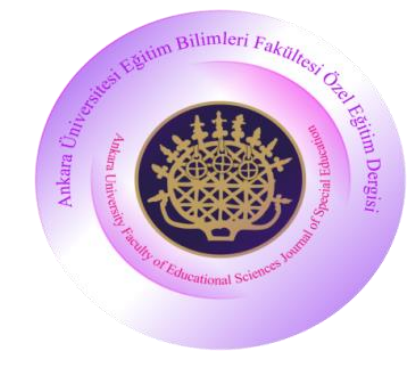

\section{Ankara University Faculty of Educational Sciences Journal of Special Education}

Year: 2018, Volume: 19, No: 1, Page No: 181-198

DOI: 10.21565/ozelegitimdergisi.279652

\title{
Preschool Inclusion Quality: Context and Assessment
}

\author{
Betül Yılmaz \\ Gazi University
}

\author{
Necdet Karasu \\ Gazi University
}

\begin{abstract}
There are several questions about the definition of inclusion, legal and educational requirements for inclusion, features of inclusive settings, and families' and teachers' current states in inclusive education framework in Turkey. In this respect, quality of inclusion may affect lots of dimensions about inclusion. Quality of preschool inclusion is important in terms of implementation and effectiveness of practices, as well as services delivery. In recent years, literature has been focused on "how" to deal with inclusion and how to make it qualified rather than the meaning of inclusion. Preschool inclusion quality, characteristics of high-quality inclusion programs and evaluation of quality issues are discussed in this article.
\end{abstract}

Keywords: Inclusion, preschool inclusion, quality, assessment of quality.

\section{Recommended Citation}

Yılmaz, B., \& Karasu, N. (2018). Preschool inclusion quality: Context and assessment. Ankara University Faculty of Educational Sciences Journal of Special Education, 19(1), 181-198. doi: 10.21565/ozelegitimdergisi.279652

*Corresponding Author:Res. Assist., Ankara, E-mail: betulyilmaz@gazi.edu.tr, https://orcid.org/0000-0002-2633-5592

${ }^{* *}$ Assoc. Prof., E-mail: necdetkarasu @ yahoo.com, https://orcid.org/0000-0001-7507-4109 
Children with special needs in their early childhood periods can receive education together with their typically developing peers in the preschool education settings within the framework of laws and regulations in Turkey. As children with special needs coexist with typically developing peers in basic education classes, it brings forward the concept of "inclusion". This conceptis shapedon the basis of the least restrictive environments (Batu and Kırcaali-İftar, 2005; Kargin, 2004; Salend, 1998).

Preschool inclusion differs from the other inclusive practices due to the fact that preschool education schools have different physical features from the other schools, and they encapsulate developmental and childcentered education programmes. As preschool children have different developmental characteristics than primary school children, the academic success-oriented system is not included in the preschool period (Wolery and Odom, 2000). Research studies have been carried out for more than thirty years have shown that it is beneficial for children with special needs to be in the same environment with their typically developing peers (Bruder, 2010; Henninger and Gupta, 2014). Inclusion practices have more positive effects on the academic performance, social development and behaviours of the children with special needs in the inclusion settings (Buysse and Bailey, 1993; Lipsky and Gartner, 1996). For instance, language development and social skills of children are improving, and they make significant progress in early literacy skills when they take part in social inclusion programmes (Green, Terry and Gallagher, 2014; Rafferty, Piscitelli and Boettcher, 2003). Furthermore, they develop positive social interactions with their peers (Hollingsworth and Buysse, 2009), and there is also an increase in the level of their social acceptance (Odom et al., 2006). Children in inclusion settings are able to easily learn communication, social and academic skills in the individualized education programmes compared to their peers who receive education in segregated settings with similar characteristics (Hunt, Farron-Davis, Beckstead, Curtis and Goetz, 1994). When it is evaluated in various aspects, the benefits of the inclusion practices for children with special needs are observedin the least restrictive environments, and the expected goals are achieved through social relationships, active participation, and increasing the learning objectives for all children (Odom, Buysse and Sokukakou, 2011). High quality environments provide more behavioral and developmental progress for children (Bryant, Burchinal, Lau and Sparling, 1994; Burchinal, Roberts, Nabors and Bryant, 1996); assessment and support of the preschool inclusion programmes and provide point of views for teachers' professional development (Soukakou, Winton, West, Sideris and Rucker, 2015; Vlachou and Fyssa, 2016) and to create preschool inclusion quality standards (Soukakou et al., 2015) are considered as significant dimensions.

As it is emphasized in the literature, contrary to the common belief, the cost of providing education in inclusion settings for children with disabilities is not more expensive than the education provided in segregated settings (Barton and Smith, 2014; Odom et al., 2001). There are many dimensions in regard to the success of inclusion, achievements provided for children and families, the services provided within the scope of inclusion, and the skills of personnel involved in inclusion. It also provides long-term and short-term benefits as well as the low-cost implementation procedures, thus these dimensions lead the countries to follow preschool inclusion. In addition, the quality of inclusion practices has often been dealt in literature as a significant issue in recent years. When studies on the quality assessment are discussed inclusively, it should be emphasized that the interest for preschool inclusion, indicators of the provided services in different dimensions, and preschool inclusion practices should be supported (Loreman, Forlin and Sharma, 2014). The dimensions of the quality of inclusion programs should be revealed for the organization, development, and evaluation of the curricula for the children with special needs and their families to benefit from inclusion at utmost level (Buysse and Hollingsworth, 2009). Examining the studies on the inclusion of children with disabilities, it is seen that one of the two directions is an assessment of the quality (Odom et al., 2011). However, the quality of preschool inclusion settings has not been a mainly focused issue in Turkey. Accordingly, the purpose of this article is to provide perspectives on this subject by presenting information on the quality of preschool inclusion, the dimensions of the quality assessment and the assessment tools that can be used for this purpose. 


\section{Quality of Preschool Inclusion}

Quality defined as a concept that has descriptive and normative features and can be dealt in many ways (Adams, 1993) according to different groups. The definition of quality changes according to the different groups; children, families, employers, and other service providers define quality in terms ofdifferent values and needs (Katz, 1993, Layzer and Goodson, 2006). Furthermore, "quality" is dealt with four differentpoints of views, including professionals, parents, children, and personnel/employees. The characteristics of the environment and status of materials are reflected through the adults' point of views; the quality is assessed by taking into account the services received by families who have a different perspective outside of the schools and the experiences of the personnel in the inclusion programmes (Katz, 1993). Nevertheless, Loreman et al. (2014), have also indicated that inclusion quality can be evaluated in micro, meso and macro levels. Micro level defines classrooms and individuals, meso level schools and macro level defines the system in which all these levels exist. Teachers in micro levels play a critical role in executing the quality of inclusion environments, and they are significant factors affecting the quality of education. In macro level, it is seen that attitudes towards inclusion, policies, the development of a shared vision, education and support services; classroom, school, organization structures and society are seen as key structures affecting preschool inclusion and quality (Buysse, Wesley, Brayant and Farner, 1999; Lieber et al., 2000; Loreman, 2007). In this context, preschool inclusion quality focuses on many various factors such as characteristics of teachers and children, program variables, teaching strategies, partnership between families and professionals, arrangements of the physical environments and materials, adapting daily routines and individualization etc. (Cate, Diendorf, McCullough, Peters and Whaley, 2010).

There are many features that couldbe dealt in the scope of preschool inclusion quality. These features are approached through dimensions such as structural quality which includes variables such as teacher-student ratio, group size, and educational status of the teacher; process quality that affects the daily needs that are directly related to teacher-child interaction, practice of the curriculum and finally the global quality or overall quality which is the combination of two dimensions (Hestenes, Cassidy, Hedge and Lower, 2007; Hestenes, Cassidy, Shim and Hedge, 2008; Phillipsen, Burchinal, Howes and Cryer, 1997). While structural quality includes regulatable features, process quality includes environmental features (Wolery and Odom, 2000). The measurement of structural quality is relatively easy as information is gathered via interviews. However, process quality requires detailed and extensive observations in different environments in a certain period of time (Ishimine and Tayler, 2014). Global quality requires being dealt holistically with the process and structural quality as well as various dimensions of quality. Structure, process and global quality features mostly reflect the researchers and professionals' perspectives on the quality. The measurement of global quality requires the evaluation of general quality indicators as well as multidimensional data collection and evaluation in accordance with the dimensions such as access, participation, and support published in the document by the institutions; The Division for Early Childhood of the Council for Exceptional Children (DEC) and The National Association for the Education of Young Children (NAEYC) in 2009 (DEC and NAEYC, 2009). Access means removing physical barriers to provide various activities and environments for children and making necessary arrangements to support their development and learning in the best way. The universal design principles are also emphasized in the access dimension both in the physical and learning environment (Barton and Smith, 2015). Participation is defined beyond the access dimension, and it creates a sense of belonging for children by keeping them available for play and learning activities and create a ground for support and high-quality inclusion practices. Furthermore, it is stated that inclusion covers the values, policies, and practices supporting the rights of every child and parent, regardless of their skills, every child has the right to participate in various activities as a member of society and all these dimensions constitutes the basis for high-quality inclusion practices in preschool period (DEC and NAEYC, 2009).

Another point that is emphasized in literature related to preschool inclusion is the preschool education influencing the quality of inclusion. The infrastructure that constitutes the assessment of the "quality of preschool inclusion" includes the studies on the quality of preschool education environments. It is seen that there have been many efforts for the preschool inclusion since the end of the 2000s. High-quality preschool education programmes 
are necessary for the quality of preschool inclusion programmes, though it is not enough. It is emphasized that the developmental needs of children with disabilities should be met in the inclusion settings (Wolery and Odom, 2000). There are limited research studies on the quality of preschool education in Turkey (Y1lmaz, 2014), yet it is noteworthy that the quality of inclusion has been recently emphasized as the main issue. It is seen that Ministry of National Education has attempted to develop the quality and standards of the preschool education and aims to create standards that support the development of all children, sensitive to the rights of education and development of children with special needs in the preschool period (Milli Eğitim Bakanlığı, 2015).

\section{Assessment of Preschool Inclusion Quality}

Quality assessment is usually donein order to improve the practices and contribute to the policy development in the scope of regulations/reviews and research within the framework of the certain approaches (Siraj-Blatchford and Wong, 1999). There are many studies in literature evaluating the quality of inclusion settings in order to ensure that children with special needs benefit from inclusion settings at utmost level, they develop models related to this and present the existing situation (La Paro, Sexton and Snyder, 1998; Hestenes et al., 2008; Fyssa and Vlachou, 2015). In order to evaluate the quality of preschool inclusion, it is seenthat many tools based on different approaches are developed (Cate et al., 2010). The need for developing these tools arose from different reasons. This need comes from;

1. evaluating long-term and short-term experiences of children in inclusion programmes to contribute to the evidence-based practices and policies;

2. program evaluations related to alternative funding and professional development;

3. self-assessment for programs in order to improve their effectiveness;

4. developing the quality standards of inclusion;

5. evaluating accountability and policy evaluation (Lero, 2010).

In this context, the most common tools and features used in the research studies for evaluating preschool inclusion quality are given below:

Early Childhood Environment Rating Scale (ECERS) is developed by Harms, Cryer and Clifford in the year of 1980 and reviewed in 2007 (ECERS-Revised), the scale is used for evaluating the preschool education programme and settings applied to children aged between 2.5-5 (Cate et al., 2010). It covers seven sub-dimensions and 43 items, including classroom, furniture, personal care routines, language and reasoning, interaction, activities, program structure, family, and staff. Although this tool evaluates the dimensions of early childhood programmes in a valid and useful way, it is not developed as a tool that covers especially the classroom practices and children with disabilities (Soukakou, 2012). However, it was used for evaluating the inclusion settings in some research studies (La Paro et al., 1998; Buysse et al., 1999; Hestenes et al., 2008). There are 35 items in sub-dimension of the latest updated ECERS, and it is used for evaluating the quality of preschool education settings of children between the ages of 3-5 (http://www.ersi.info/ecers3.html). This scale which has been translated into several languages and is widely used has also been used in Turkey to investigate the quality of early childhood education settings (Aksoy, 2009; Feyman, 2006; Göl-Güven, 2009; Güçhan-Özgül, 2011; Kalkan, 2008; Tekmen, 2005; Solak, 2007). Although it has some limitations, itis seen as a useful evaluation tool (Ishimine and Tayler, 2014). There is also another version of the scale for evaluating the environment in which young children up to 30 months are included. It is called "Infant / Toddler Environment Rating Scale (ITERS)" and has seven sub-dimensions and 39 items, including classroom and furniture, personal care and routines, listening and speaking, activities, interaction, program structure, family, and personnel. The use of this tool requires training by the experts. 
The Quality Inclusive Experiences Measure (QuIEM) is developed by Wolery, Pauca, Brashers and Grant in 2000 so in order to evaluate the experiences of children with special needs in the inclusion settings (as cited in Soukakou, et al 2015). The objectives of the programme implemented in education environment are within the scope of seven dimensions and include the support of institution employees, adequacy, and accessibility of the physical environment, participation, adult-child communication and interaction, individualization and child-child communication and interaction. This scale provides information on the general quality of the inclusion and the experiences of a child who received inclusive education by taking a sample of the time and observing it by recording the event, interviewing with the employees in institutions and making the review of the documents. It is emphasized that there isa need for more research results to validate the use of QuIEMin order to measure the quality of inclusion programmes (Odom et al., 2011). It is indicated that there is a need for conducting more research studies on the psychometric features of the scale in the scope of availability and reliability of it (Fyssa and Vlachou, 2015; Spiker, Hebbeler and Barton, 2011).

The SpeciaLink Early Childhood Inclusion Quality Rating Scale (SECIQR) which is createdon the basis ofSpeciaLink Child Care Inclusion Practices Profile and Principles Scale developed by Irwin in 2005 (as cited in Lero, 2010; Soukakou, 2012). This scale is finalized in 2009 and consists of two parts; practices and principles (http://www.specialinkcanada.org/about/history.html). While the practices section consists of 11 items and 158 indicators such as physical setting, education of the institution employees, therapies, preparation for the school system and materials, and so forth, the principles section consist of 6 items and 92 indicators such as full participation, leadership, zero rejection, leadership and precautionary principles. The inclusion quality is analysed by collecting data through observation, document review, and interviews (Lero, 2010). This scale evaluates the long-term and short-term effects of the inclusive practices on children and contributes to the evidence-based policies and practices. It provides information on the development of quality standards, used for developing models that can support the professional development by evaluating programmes (Cate et al., 2010). Like ECERS, each item is scored with seven grades; there are 250 special indicators in 17 items (Spiker et al., 2011). The collected data by the help of this scale provides information on the structure quality rather than the general quality of the inclusion settings. It is indicated that the psychometric features of this scale should be supported with more research studies (Soukakou, 2012).

Inclusive Classroom Profile (ICP) is applied in classrooms in which preschool inclusion practices are conducted with children between the ages of $2-5$, and at least one child has been diagnosed with a developmental disability. A pilot study was conducted in England to determine the validity and reliability of the scale, and then psychometric properties were tested in different studies (Soukakou, 2012; Soukakou, Winton and West, 2012; Soukakou et al., 2015). There are 12 items in total including; adaptation of space, materials, and equipment, adult involvement in peer interaction, adult guidance of children play, conflict resolution, membership, relationship between adults and children, support for communication, adaptation of group activities, transition between activities, feedback, family-professional partnerships, and monitoring children's learning in ICP. The operational definition of each item is explained, and the features related to the scoring are clarified separately. ICP is a likert type scale scored in seven grades, 1 point for inappropriate and harmful applications and 7 points for the applications that are considered to be beneficial for supporting individualization in inclusion. Developed in the United Kingdom and used in the United States, ICP has been developed to be adapted to the other countries as well (Soukakou, 2012). It can be used with the aim of professional development programme analysis and policy development as well as providing information on the quality of preschool inclusion settings (Soukakou et al., 2015). Its use requires standard training process and certificate. The tool was first translated into Turkish by Yllmaz (2014), and several attempts were made to determine the quality of inclusion classrooms in the independent kindergartens and private preschools in Ankara.

Quality Rating and Improvement System (QRIS) is used to assess the quality of early childhood education programmes in several states in the USA, to increase the demands of the families for high-quality early childhood education programmes, and to provide professional development in educational service providers (Cate 
et al., 2010). This system is usedin order to evaluate the quality of many state programmes, to monitor the quality and development of these programmes and provide information to families and other parties related to this. The QRIS system used by some of the states consists of performance standards in regard to children with disability and their families. When these standards are examined, it is seen that different dimensions of inclusion are emphasized on the basis of these standards (i.e., adaptations, activities intended for individualized education plans, professional development, and so forth), though there is no agreement on the overall inclusion quality programme (National Professional Development Center on Inclusion [NPDCI], 2009). QRIS is used effectively to establish standards for the early childhood and quality programmes in preschool inclusion conducted in the USA.

In short, it is noteworthy that the tools whichare expressed as having strengths and weaknessesin terms of their psychometric features, provide data for multidimensional evaluation, especially the general qualitative evaluation through observation, interviews and document review. ECERS is among the most widely used tools in evaluating preschool environments; itis stated that with the latest updates it is used for evaluating the preschool inclusion environments. Compared to the other tools, QuIEM provides limited data, the findings supporting the research should be revealed. SECIQR informs more about the structural quality and differs from the other tools in terms of categorizing the items. ICP being a new and current scale provides a more extensive point of view in terms of taking into account the literature in the dimension of preschool quality in terms of DEC and NAEYC criteria (Soukakou, 2012). Moreover, it could be used not only for quality evaluation but also for professional development, monitoring, and policy development. It is via the reflection of the usage of QRIS system that exemplifies the evaluation of the program quality and also monitors their developments as well as contributing to the formation of standards and enabling to be used in the region base of the tools for quality evaluation. However, there is no system for evaluating the quality of preschool inclusion, so it canbe stated that QRIS is not only the widespread system but also it provides limited data.

\section{Conclusion and Implications}

This article aims to bring forward the information which can be used as a starting point for quality assessment and the assessment tools used in regard to the "preschool inclusion quality" that is frequently dealt in literature in recent years. The preschool inclusion is a field that requires the provision of significant services for the families and children with developmental disabilities in their early childhood and provides benefit for both children with special needs and typically developing children (Henninger and Gupta, 2014). In order to increase the service quality provided in this field and to produce high-quality inclusion practices, firstly it is necessary to present the required dimensions and features of the "quality." It is the feature of quality practices that make the inclusion practices qualified rather than the fact that students with developmental disabilities are in the same environment with the typically developing children. The quality in preschool inclusion covers many structural and procedural features such as the classes of children with disabilities, interaction in these classes; from teachers' experiences and their wages to their cooperation with the parents, and so forth. There are different scales to measure these features. The professional development of these tools and their use in research studies are seen as a key factor for high-quality preschool inclusion practices. Furthermore, it is suggested that in the preschool inclusion access level; the universal design principles and supporting technologies should be used for learning, in participation level; embedded education, natural education methods, and other supportive strategies should be in classrooms, in support level; studies should be carried out related to professional development, cooperation models and family-professional partnership (NPDCI, 2011). In the context of inclusion practices in Turkey, it is considered that studies should be carried out in regard to access, participation and support level so as to prove how preschool inclusion will be effective. Moreover, considering the developments related to "preschool inclusion quality" and "preschool education quality," it is seen that there is a need for conducting more studies within the scope of both fields in Turkey. Additionally, it is significant to carry out studies to present the relationship between preschool inclusion quality and dimensions related to process quality, structural quality, and general quality features. It is indicated that the barriers against the high-quality preschool inclusion settings are related to the cooperation among personnel training, preschool education, and special trainers (Odom and Bailey, 2001). From this point of view, it 
is noteworthy that there is a need for research studies that examine the influence of different studies on teachers' professional development on the quality of preschool inclusion. It is important to integrate these practices into the system and spread its area as well to integrate high-quality inclusion practices. At this point, it is considered to be beneficial that information received by the assessment tools collected in the data based system to develop the quality standards are meaningful and beneficial for children in preschool inclusion settings, their teachers, and families. 